\title{
Aislamiento de Plesiomonas shigelloides y Aeromonas veronii biotipo sobria en heces de lobo marino común sudamericano, Otaria flavescens (Shaw, 1800)
}

\author{
Isolation of Plesiomonas shigelloides and Aeromonas veronii biotype sobria from \\ South American sea lion, Otaria flavescens (Shaw, 1800) feces \\ Mario J. González', María P. Villanueva', Fadua Latif ${ }^{1}$, \\ Fabiola Fernández ${ }^{1}$ y Heriberto Fernández ${ }^{1}$ \\ ${ }^{1}$ Instituto de Microbiología Clínica, Universidad Austral de Chile, Casilla 567, Valdivia, Chile \\ hfernand@uach.cl
}

\begin{abstract}
Plesiomonas shigelloides and Aeromonas spp. are Gram negative bacteria vastly distributed in the environment, being isolated from aquatic ecosystems and terrestrial and marine animals. The South American sea lion (Otaria flavescens) is the most frequent marine mammal of the Chilean coasts, living in beaches, rocks or coastline of rivers. In this work we determined the isolation frequency of $P$. shigelloides and A. veronii biotype sobria in fecal samples of South American sea lions belonging to a colony established at the urban South coastline of Valdivia River, southern Chile.
\end{abstract}

\section{Introducción}

Plesiomonas shigelloides y Aeromonas sp. son bacilos Gram negativos, anaerobios facultativos y oxidasa positivos. El género Plesiomonas, con su única especie $P$. shigelloides, sufrió un cambio taxonómico en el año 2001, siendo trasladada desde la familia Vibrionaceae a la familia Enterobacteriaeae (González-Rey et al. 2004). El género Aeromonas, con 15 especies reconocidas actualmente, pertenece a la familia Aeromonadaceae (Huddleston et al. 2006). P. shigelloides y Aeromonas spp. habitan ecosistemas acuáticos, siendo además, aisladas de una amplia variedad de animales. Aeromonas spp. ha sido aislada de peces, moluscos, reptiles, delfines, belugas y lobo marino californiano. $P$. shigelloides ha sido aislada de peces, aves acuáticas (cisnes, cigüeñas, gaviotas), mamíferos terrestres (perros, gatos, zorros) y mamíferos marinos (belugas y lobo marino californiano) (Sugita et al. 1995, Rahman et al. 2002, González-Rey et al. 2004, Moreno et al. 2006, Pereira et al. 2004, Higgins 2000, Johnson et al. 2006).

El lobo marino común sudamericano (Otaria flavescens Shaw, 1800) es el mamífero marino más frecuente en las costas chilenas. Su distribución geográfica es amplia y comprende desde el norte del Perú
From the 30 samples under study, $P$. shigelloides was isolated in $27(90.0 \%)$ and A. veronii biotype sobria in 17 (56.6\%). To our knowledge, this is the first report of the isolation of $P$. shigelloides and Aeromonas spp. from South American sea lions in Chile. However, further studies are needed to clarify if these bacteria play any role in producing disease, or are merely commensals, in these marine mammals.

Key words: Aeromonadaceae, Enterobacteriaceae, intestinal microbiota, marine mammals, reservoir

hasta el Cabo de Hornos por el lado del Océano Pacífico y alcanzando, por el Océano Atlántico, hasta el sur de Brasil (Sielfeld 1999). Esta especie se congrega en sitios costeros denominados loberas, las cuales pueden estar ubicadas en farellones, requeríos o playas. Su alimentación consiste en peces, crustáceos y moluscos, los que son obtenidos directamente del ecosistema marino. Algunas poblaciones de lobos marinos pueden establecer una interacción con el hombre, compitiendo directamente por el recurso o alimentarse de los desechos que el hombre no utiliza (Pavés et al. 2005, Sielfeld 1999). El ingreso del lobo marino común al río Valdivia y sus afluentes $\left(39^{\circ} 47^{\prime} \mathrm{S}, 73^{\circ} 15^{\prime} \mathrm{W}\right)$ fue documentado científicamente en 1976 y la penetración de este animal a este sistema fluvial obedecería a la búsqueda de alimento (Schlatter 1976).

El objetivo de este estudio fue determinar la presencia de $P$. shigelloides y Aeromonas spp. en heces del lobo marino común sudamericano establecido en la parte urbana de la ribera sur del río Valdivia, Chile.

\section{Material y métodos}

Se estudió una colonia de lobos marinos constituida por 38 ejemplares, situada en la ribera del río Valdivia. Desde 
el sitio de descanso de los animales, fueron recolectadas 30 muestras fecales en recipientes estériles y remitidas al laboratorio para análisis bacteriológico. Se tomaron 5 $\mathrm{g}$ de heces de cada muestra y se depositaron en frascos con $50 \mathrm{ml}$ de agua peptonada alcalina (APA), $\mathrm{pH} \mathrm{8,6}$ (caldo de enriquecimiento) y se incubaron a $37^{\circ} \mathrm{C}$ por 6 h. Posteriormente, alícuotas del caldo de enriquecimiento fueron sembradas en placas de agar sangre ampicilina $\left(10 \mu \mathrm{g} \mathrm{mL}^{-1}\right)$, agar sangre y agar Mc Conkey, las cuales fueron incubadas a $37^{\circ} \mathrm{C}$ por $24 \mathrm{~h}$. A las colonias sospechosas de Aeromonas sp. se les realizó la prueba de oxidasa, producción de ácido a partir de carbohidratos (arabinosa, lactosa, sacarosa, manitol, inositol, salicina), descarboxilación de lisina y ornitina, arginina dihidrolasa, hidrólisis de esculina, indol, susceptibilidad a cefalotina, ampicilina y susceptibilidad al agente vibriostático $\mathrm{O} /$ $129(10 \mu \mathrm{g}$ y $150 \mu \mathrm{g})$ (Castro-Escarpulli et al. 2003). Para $P$. shigelloides se realizó la prueba de oxidasa, fermentación de la glucosa, crecimiento en $\mathrm{NaCl}$ al $3 \%$ y al 6\%, susceptibilidad al agente vibriostático O/129 (10 $\mu \mathrm{g}$ y $150 \mu \mathrm{g}$ ) y producción de ácido a partir de inositol (Castro-Escarpulli et al. 2003).

\section{Resultados y discusión}

La Tabla 1 indica que de las 30 muestras recolectadas, $27(90,0 \%)$ fueron positivas para $P$. shigelloides y 17 (56,6\%) para A. veronii biotipo sobria. En la literatura no existen estudios relacionados con la microbiota intestinal del lobo marino común sudamericano en su medio natural. Sugita et al. (1996) caracterizaron la microbiota intestinal de cinco ejemplares de lobo común sudamericano confinados en el 'Enoshima Aquarium', localizado en Kanagawa, Japón, aislando Aeromonas spp. en los cinco ejemplares, sin establecer la identificación a nivel de especie, no obstante $P$. shigelloides no fue aislada de estos ejemplares.

La microbiota del lobo marino californiano (Zalophus californianus) ha sido estudiada con más detalle. Hernández-Castro et al. (2005) caracterizaron la microbiota aeróbica de la cavidad nasal de 57 crías de lobo marino californiano, aislando Aeromonas spp. en una muestra $(1,75 \%)$. Por otro lado, Johnson et al. (2006) realizaron un estudio sobre la microbiota aeróbica de prepucio y vagina de la misma especie de lobo marino, en animales salvajes y en animales enfermos mantenidos en cautiverio. Considerando los dos grupos de animales bajo estudio, adultos en estado salvaje $(n=65)$ y en cautiverio $(\mathrm{n}=35), P$. shigelloides fue aislada solamente en una muestra (1,5\%) del primer grupo. En el segundo grupo, Aeromonas spp. fue aislada en cuatro muestras $(11,4 \%)$ y $A$. hydrophila en tres $(8,6 \%)$.

En los estudios anteriormente señalados, el porcentaje de aislamiento $P$. shigelloides y de Aeromonas spp. fue menor en comparación a nuestros resultados, donde alcanzó al 90,0\% y el 56,6\% respectivamente de las muestras estudiadas. Por otro lado, A. veronii biotipo sobria no fue aislada de los ejemplares de lobo californiano estudiados (Hernández-Castro et al. 2005, Johnson et al. 2006). La permanencia de los lobos marinos en las riberas del rio Valdivia, podría haber favorecido la proliferación de A. veronii biotipo sobria y $P$. shigelloides, ya que el rio representaría un hábitat adecuado para estas bacterias (González-Rey et al. 2004, Huddleston et al. 2006). Otra ruta de transmisión y posterior colonización, sería el consumo de los desechos de pescados y mariscos, los cuales son eliminados de una feria libre (Moreno et al. 2006). Para establecer fehacientemente si estas especies bacterianas formarían parte de la microbiota de O. flavescens, es necesario realizar estudios longitudinales en colonias de mayor tamaño, establecidas en sitios alejados de la actividad humana. Al estudiar la microbiota de colonias no relacionadas con las actividades humanas, se podría obtener información para comparar y esclarecer si estas bacterias, como las encontradas en nuestro estudio, son transmitidas por el contacto con el medio acuático (río) y/o la ingesta de productos del mar contaminados.

\section{Tabla 1}

Frecuencia de aislamiento de Plesiomonas shigelloides y Aeromonas veronii biotipo sobria de heces de lobo marino común sudamericano (O. flavescens)

Isolation of Plesiomonas shigelloides and Aeromonas veronii biotype sobria from feces of South American sea lion (O. flavescens)

\begin{tabular}{llc}
\hline \multicolumn{1}{c}{ Especie } & $\mathrm{N}^{\circ}$ muestras estudiadas / $\mathrm{N}^{\circ}$ muestras positivas & $\%$ \\
\hline P. shigelloides & $30 / 27$ & 90,0 \\
A. veronii biotipo sobria & $30 / 17$ & 56,6 \\
\hline
\end{tabular}




\section{Agradecimientos}

Este trabajo fue financiado por el proyecto DID-UACH S-2007-37.

\section{Literatura citada}

Castro-Escarpulli G, MGAguilera-Arreola, CH HernándezRodríguez, RI Arteaga-Girabay, AA CarmonaMartínez, A Pérez-Valdespino, S Giorgo-Cerezo, MJ Figueras \& G Aparicio. 2003. La identificación genética de Aeromonas, una realidad y una necesidad para la microbiología diagnóstica. Bioquimia 28(4): 11-18.

González-Rey C, S Svenson, L Bravo, A Siitonen, V Pasquale, S Dumontet, I Ciznar \& K Krovacek. 2004. Serotypes and anti-microbial susceptibility of Plesiomonas shigelloides isolates from humans, animals and aquatic environments in different countries. Comparative Inmunology, Microbiology \& Infectious Diseases 27: 129139.

Hernández-Castro R, L Martinez-Chavarria, A Diaz-Avelar, A Romero-Osorio, C Godinez-Reyes, A Zavala-González \& A Verdugo-Rodriguez. 2005. Aerobic bacterial of the nasal cavity in gulf of California sea lion (Zalophus californianus) pups. Veterinary Journal 170: 359-363.

Higgins R. 2000. Bacteria an Fungi of marine mammals: A review. Canadian Veterinary Journal 41: 105-116.

Huddleston J, J Zak \& R Jeter. 2006. Antimicrobial susceptibilities of Aeromonas spp. isolates from environmental sources. Applied and Environmental Microbiology 72: 7036-7042.

Johnson S, L Lowenstine, F Gulland, S Jang, D Imai, F Almy, R DeLong \& I Gardner. 2006. Aerobic bacterial flora of the vagina and prepuce of California sea lions (Zalophus californianus) and investigation of associations with urogenital carcinoma. Veterinary Microbiology 114: 94103.
Moreno M, L Medina, J Álvarez, J Obregón \& G Medina. 2006. Detección de Plesiomonas shigelloides mediante la PCR en tilapias silvestres (Oreochromis mossambicus) y cultivadas (Tetrahíbrido O. mossambicus x O. urolepis hornorum x O. niloticus x $O$. aureus) en Venezuela. Revista Científica, Facultad de Ciencias Veterinarias, Universidad del Zulia 16(5): 459-465.

Pavés H, R Schlatter \& C Espinoza. 2005. Patrones reproductivos del lobo marino común, Otaria flavescens (Shaw 1800), en el centro-sur de Chile. Revista Chilena de Historia Natural 78: 687-700.

Pereira C, C Possas, C Viana \& D Rodrigues. 2004. Aeromonas spp. e Plesiomonas shigelloides isoladas a partir de mexilhões (Perna perna) in natura e pré-cozidos no Rio de Janeiro, RJ. Ciência e Tecnologia de Alimentos, Campinas 24(4): 562-566.

Rahman M, P Colque-Navarro, I Kühn, G Huys, J Swings \& R Möllby. 2002. Identification and characterization of pathogenic Aeromonas veronii biovar Sobria associated with epizootic ulcerative syndrome in fish in Bangladesh. Applied and Environmental Microbiology 68: 650-655.

Schlatter R. 1976. Penetración del lobo marino común, Otaria flavescens Shaw, en el río Valdivia y afluentes. Medio Ambiente 2(1): 86-90.

Sielfeld W. 1999. Estado del conocimiento sobre conservación y preservación de Otaria flavescens (Shaw, 1800) y Arctocephalus australis (Zimmermann, 1783) en las costas de Chile. Estudios Oceanológicos 18: 81-96.

Sugita H, K Tanaka, M Yoshinami \& Y Deguchi. 1995. Distribution of Aeromonas species in the intestinal tracts of river fish. Applied and Environmental Microbiology 61: 4128-4130.

Sugita H, Y Takanashi, Y Sasaki, T Nishina, E Aono, A Fujimoto \& Y Deguchi. 1996. The intestinal microflora of sea lions reared in a aquarium. Fisheries Science 62: 321-322. 